\title{
Erratum to: Hydrophilic molecularly imprinted polymers for bisphenol A prepared in aqueous solution
}

\author{
Naoko Inoue • Tooru Ooya • Toshifumi Takeuchi
}

Published online: 14 August 2014

(C) Springer-Verlag Wien 2014

Erratum to: Microchim Acta (2013) 180: 1387-1392

DOI 10.1007/s00604-013-0996-5

The original version of this paper, unfortunately, contained an error.

The corresponding author's name was incorrectly captured. The correct name of the corresponding author is Toshifumi Takeuchi (first name/last name).

The online version of the original article can be found at http://dx.doi.org/ 10.1007/s00604-013-0996-5.

N. Inoue $\cdot$ T. Ooya $\cdot$ T. Takeuchi $(\bowtie)$

Graduate School of Engineering, Kobe University, 1-1

Rokkodai-cho, Nada-ku, Kobe 657-8501, Japan

e-mail: takeuchi@gold.kobe-u.ac.jp

$\mathrm{N}$. Inoue

Center for Collaborative Research and Technology Development,

Kobe University, 1-1 Rokkodai-cho, Nada-ku, Kobe 657-8501,

Japan 